\title{
Chinese herbal bath therapy for the treatment of uremic pruritus: meta-analysis of randomized controlled trials
}

\author{
Wenxuan Xue, Yanhua Zhao, Mengyun Yuan and Zhiqiang Zhao*
}

\begin{abstract}
Background: Chinese herbal bath therapy (CHBT) is a traditional external therapy that has been used for the treatment of uremic pruritus (UP) in China. We conducted a meta-analysis to evaluate the efficacy and safety of CHBT for UP.

Methods: We searched seven databases for studies published since database inception to September 1, 2018. Randomized trials evaluating CHBT for UP were collected. The therapeutic effects of CHBT were measured by the pruritus level (via the visual analogue scale (VAS) or the symptom score scale) and the total effective rate. We combined studies using mean difference (MD) for continuous outcomes and using risk ratio for dichotomous data, both with 95\% confidence intervals. RevMan V.5.3 software was used to assess the data reported and perform the meta-analysis.

Results: Seventeen articles including 970 patients were identified. All participants were haemodialysis (HD) patients. CHBT is administered by immersing the whole body in a prepared herbal water bath. On average, an herbal bath prescription included 11 Chinese herbs. The mean treatment duration was 4.7 weeks. Compared with basic treatment (HD or haemoperfusion (HP)) and adding a control of sham CHBT, clear hot water bath, or calamine lotion, CHBT plus basic treatment reduced the VAS score $(\mathrm{MD}=-2.38 ; 95 \%$ confidence intervals $[\mathrm{CI}],-3.02$ to $-1.74 ; P<0.00001)$ and the symptom score $(\mathrm{MD}=-8.42 ; 95 \%$ confidence intervals $[\mathrm{Cl}],-12.47$ to $-4.36 ; P<0.00001)$ and had a higher total effectiveness rate (risk ratio $[\mathrm{RR}]=1.46 ; 95 \% \mathrm{Cl}, 1.31$ to $1.63 ; P<0.00001$ ).
\end{abstract}

Conclusions: In conclusion, CHBT could be a complementary therapy for improving pruritic symptoms in uraemia patients. More rigorously designed, multicentre, prospective RCTs are warranted to further identify the efficacy and safety of CHBT.

Trial registration: Systematic review registration: [PROSPERO registration: CRD42018108506].

Keywords: Bath, Uremic pruritus, ESRD, Haemodialysis, Traditional Chinese medicine

\section{Background}

Uremic pruritus (UP) is one of the most common and troublesome symptoms in patients with chronic renal failure and occurs in $46 \%$ of haemodialysis patients [1-3]. UP causes great distress to patients and affects their mood and sleep quality. In addition, UP has an independent relationship with mortality, which is an urgent problem to solve [4-6].

\footnotetext{
* Correspondence: zzq11142017@163.com

The First College of Clinical Medicine, Nanjing University of Chinese

Medicine, 138 Xianlin Avenue, Qixia District, Nanjing City, Jiangsu Province, China

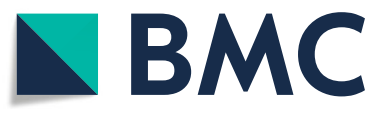

(c) The Author(s). 2019 Open Access This article is distributed under the terms of the Creative Commons Attribution 4.0 International License (http://creativecommons.org/licenses/by/4.0/), which permits unrestricted use, distribution, and reproduction in any medium, provided you give appropriate credit to the original author(s) and the source, provide a link to the Creative Commons license, and indicate if changes were made. The Creative Commons Public Domain Dedication waiver (http://creativecommons.org/publicdomain/zero/1.0/) applies to the data made available in this article, unless otherwise stated. and cromolyn sodium (a mast cell stabilizer) [12, 13], have
} and likely multifactorial. Skin or nerve inflammation [6, 7], subclinical or overt uremic neuropathy in the context of chronic systemic inflammation associated with renal failure, or increased $\mu$-opioid receptor activity due to kidney failure have all been related $[3,8,9]$.

UP is often difficult to eradicate, although the symptoms can usually be mitigated. Current medications including gabapentin (a modulator of excitatory neurotransmitters) [10], capsaicin (a mediator of substance P release) [11], 
been suggested, but no specific, effective therapies are currently available for uremic pruritus $[1,3]$.

As an ancient therapeutic method, Chinese herbal bath therapy (CHBT) has been used for thousands of years in China $[14,15]$. In the early published Chinese medical works, such as "Prescriptions for fifty-two diseases" (202 B.C.-9 A.D.) and "Treatise on Cold Pathogenic and Miscellaneous Diseases" (200 A.D.- 210 A.D.), herbal bath therapy was frequently used [15]. Importantly, herbal bath therapy is still very popular in Asian countries today and is widely used to treat skin diseases, osteoarthritis diseases and some internal diseases, of which pruritus is especially responsive [16]. While traditional Chinese medicine doctors use herbal baths to relieve itching in patients with uraemia, the mechanism of the effect is not particularly clear $[14,17]$.

To evaluate the efficacy and effectiveness of Chinese herbal bath therapy scientifically, we reviewed the medical literature comprehensively and conducted a meta-analysis of randomized controlled trials of herbal bath therapy focusing on the treatment of uremic pruritus.

\section{Methods}

\section{Protocol and registration}

This study was registered in the Preferred Reporting Items for Systematic Review and Meta-analyses Statement (PRISMA) under the registration number: CRD42018108506 (available from: http://www.crd.york.ac.uk/PROSPERO/display_record.php? ID = CRD42018108506).

\section{Search strategy}

We searched PubMed, Cochrane Library, Embase, Chinese National Knowledge Infrastructure (CNKI), Wanfang Databases, Chinese Science and Technology Periodical Database (VIP) and Chinese Biomedical Database (CBM) from their inception to September 1, 2018. The search strategy for PubMed was: Search $)((()((()(()(()(()(()(()(()(()($ Kidney Failure, Chronic"[Mesh]) OR chronic kidney failure) OR chronic kidney disease) OR chronic kidney insufficiency) OR chronic nephropathy) OR chronic renal disease) OR chronic renal failure) OR kidney chronic failure) OR kidney disease, chronic) OR kidney failure, chronic) OR kidney function, chronic disease) OR renal insufficiency, chronic) OR End-Stage Kidney Disease) OR Disease, End-Stage Kidney) OR End Stage Kidney Disease) OR Kidney Disease, End-Stage) OR End-Stage Renal Disease) OR Disease, End-Stage Renal) OR End Stage Renal Disease) OR Renal Disease, End-Stage) OR Renal Disease, End Stage) OR Renal Failure, End-Stage) OR End-Stage Renal Failure) OR Renal Failure, End Stage) OR Renal Failure, Chronic) OR ESRD)) OR $\quad(((()(()(()(()(()(()(()(()(()(()$ (c)enal Dialysis”[Mesh]) OR Dialyses, Renal) OR Renal Dialyses) OR Dialysis, Renal) OR Hemodialyses) OR Dialysis, Extracorporeal) OR Dialyses, Extracorporeal) OR Extracorporeal Dialyses) OR
Extracorporeal Dialysis) OR hemodialysis) OR blood dialysis) OR chronic haemodialysis) OR chronic hemodialysis) OR chronic intermittent haemodialysis) OR chronic intermittent hemodialysis) OR dialysis center) OR dialysis, blood) OR extracorporeal blood cleansing) OR haemodialysis) OR haemodialysis center) OR haemodialysis centre) OR haemodialysis department) OR haemodialysis unit) OR haemodialysis units, hospital) OR hemodialyse) OR hemodialysis center) OR hemodialysis department) OR hemodialysis unit) OR hemodialysis units, hospital) OR hemorenodialysis) OR hemotrialysate) OR intermittent chronic haemodialysis) OR intermittent chronic hemodialysis) OR intermittent haemodialysis) OR intermittent hemodialysis) OR kidney dialysis)) OR $(((()(($ "Peritoneal Dialysis”[Mesh]) OR Dialyses, Peritoneal) OR Dialysis, Peritoneal) OR Peritoneal Dialyses) OR intermittent peritoneal dialysis) OR peritoneal dialysis, intermittent) OR peritoneum dialysis) OR peritoneum inigation))) AND (((“"Pruritus"[Mesh]) OR Pruritis) OR Itching) OR itch)) AND (((()((((“Baths”[Mesh]) OR Bath) OR bathing) OR bathroom) OR baths) OR hydroelectric bath) OR water bath) OR waterbath) OR wash) OR washing). A similar strategy was applied to the Cochrane Library and Embase. These search terms were adapted and used in the Chinese databases with no limitations.

\section{Eligibility criteria}

Studies were included for analysis if they met the following criteria: (1) The included studies were randomized controlled trials (RCTs) recruiting participants with UP. (2) For the types of interventions, the participants of the treatment groups were given a herbal bath with only the head remaining out of the water. (3) The included studies evaluated the efficacy or effectiveness of CHBT for UP and had clear outcome data, such as total effectiveness rate or symptom scores.

The exclusion criteria were as follows: (1) The CHBT group used other traditional Chinese medicine treatment (OTCMT) methods (The control group combined with OTCMT is not excluded). (2) The details of the trials were unclear, or the outcome data were not complete.

\section{Selection of studies}

The two authors (WX and YZ) conducted the literature screening independently. The titles and abstracts of all records were first filtered for relevance, and then the full text of potentially relevant studies was identified for eligibility. If a disagreement arose, it is resolved through discussion with a third author (MY).

The effects of herbal bath therapy on the clinical symptoms of UP were measured by the pruritus level (via the visual analogue scale (VAS) or the symptom score scale) and the total effective rate. The definitions 
of the outcome measures are described in detail in Table 1.

\section{Data extraction}

Two authors (WX and YZ) extracted the following data independently using a data extraction table designed prior to initiation of the literature search: publication information, study setting, study population, age, sample size, characteristics of intervention and control, outcome indicators, and relevant indicators of bias risk assessment. The main data are summarized in Table 1 and Table 2.

\section{Statistical analysis}

We utilized Review Manager (Version 5.3, The Cochrane Collaboration, 2014) for data synthesis and analysis. For continuous outcomes, we analysed studies using mean difference (MD) with a 95\% confidence interval. MD was calculated by subtracting after from before measurements, and standard deviation (SD) for change was estimated by the given SD values before and after treatment. In addition, for dichotomous data, we combined studies using a risk ratio to compare intervention and control groups with a 95\% confidence interval. As the outcomes of this meta-analysis, VAS and Dirk R Kuypers score (DRKS) were presented as MD, while a total effectiveness rate was presented as RR. Due to significant clinical heterogeneity, we used a random-effects model for pooling. Heterogeneity was evaluated statistically using the Cochran Q statistic and the $I^{2}$ index. We used funnel plots to evaluate the potential publication bias.

\section{Methodological quality}

Two authors (WX and MY) independently appraised the methodological quality of the included studies according to the Cochrane Collaboration's risk of bias tool [18]. Any discrepancy between the investigators was resolved by the third author (YZ). The following 7 aspects were assessed: (1) random sequence generation; (2) allocation concealment; (3) patient blinding; (4) assessor blinding; (5) incomplete outcome data; (6) selective reporting; and (7) other sources of bias. The evaluation results were given a ranking of low risk, unclear risk, or high risk according to the above criteria.

\section{Results}

\section{Search results}

We searched 623 articles from 7 electronic databases according to the search strategy. A total of 308 duplicate articles were excluded. After initially screening the titles and abstracts, 235 articles were excluded because they did not fit the inclusion criteria. We retrieved 80 full-text studies for further identification, and 63 articles were removed with reasons. Finally, 17 eligible RCTs [19-35] (For links to the articles, see Additional file 1) and 970 participants were included in the meta-analysis (Fig. 1).

\section{Study characteristics}

The basic characteristics of the 17 RCTs are shown in Table 1, and the details of each research scheme are shown in Table 2. All of the RCTs were conducted and published in China. A total of 970 patients with UP were enrolled, with the sample size ranging from 24 to 156 . All participants were on haemodialysis and were undergoing basic treatment, such as controlling blood pressure, treating anaemia, or maintaining water electrolyte and acid-base balance. All of the patients were adults over the age of 18. The mean treatment duration was 4.7 weeks.

On average, an herbal bath prescription in the intervention groups included 11 Chinese herbs, ranging from 6 to 22 . In these prescriptions, two [30, 32] used granules of traditional Chinese medicine as raw materials to replace herbs. The relationship among English names, Latin names and Chinese PinYin names of the 19 most frequently used Chinese herbs are listed in Table 3. Eight Chinese herbs (Fructus Kochiae, Lightyellow Sophora Root, Densefruit Pittany Root-bark, Cicada Slough, Common Cnidium Fruit, Chinese Honeylocust Spine, Puncturervine Caltrop Fruit, and Tuber Fleeceflower Root) were described to relieve pruritus. Five Chinese herbs (Chinese Angelica, Manchurian Wildginger, Szechuan Lovage Rhizome, Suberect Spatholobus Stem, and Dan-shen root) were described to activate blood circulation. Four Chinese herbs (Chinese Ephedrs Herb, Fineleaf Schizonepeta Herb, Angelica Dahurica, and Divaricate Saposhniovia Root) were described to stimulate sweat. Two Chinese herbs, Rhubarb Root and Glabrous Greenbrier Rhizome, were described to promote detoxification [36-38]. The control groups used modern basic medical treatment, some of which were combined with sham $\mathrm{CHBT}$, clean hot water bath, or calamine lotion. Treatment duration in the RCTs ranged from 2 weeks to 3 months, and outcomes were evaluated at the end of treatment.

\section{Meta-analysis}

In the 17 eligible RCTs, three trials measured pruritus via the VAS score and five trials used the Dirk R Kuypers score, whereas 12 trials assessed clinical improvement by total effectiveness rate. Three trials measured and evaluated pruritus score and total effectiveness rates simultaneously. In addition, in three of these RCTs [28, 33, 35], no antipruritic drugs or HP were used in the control group, primarily reflecting the efficacy of $\mathrm{CHBT}$ in the treatment of UP. The 14 other RCTs using antipruritic treatments in the control group were more focused on which treatments were more effective, primarily reflecting the effectiveness of CHBT in the treatment of UP. 


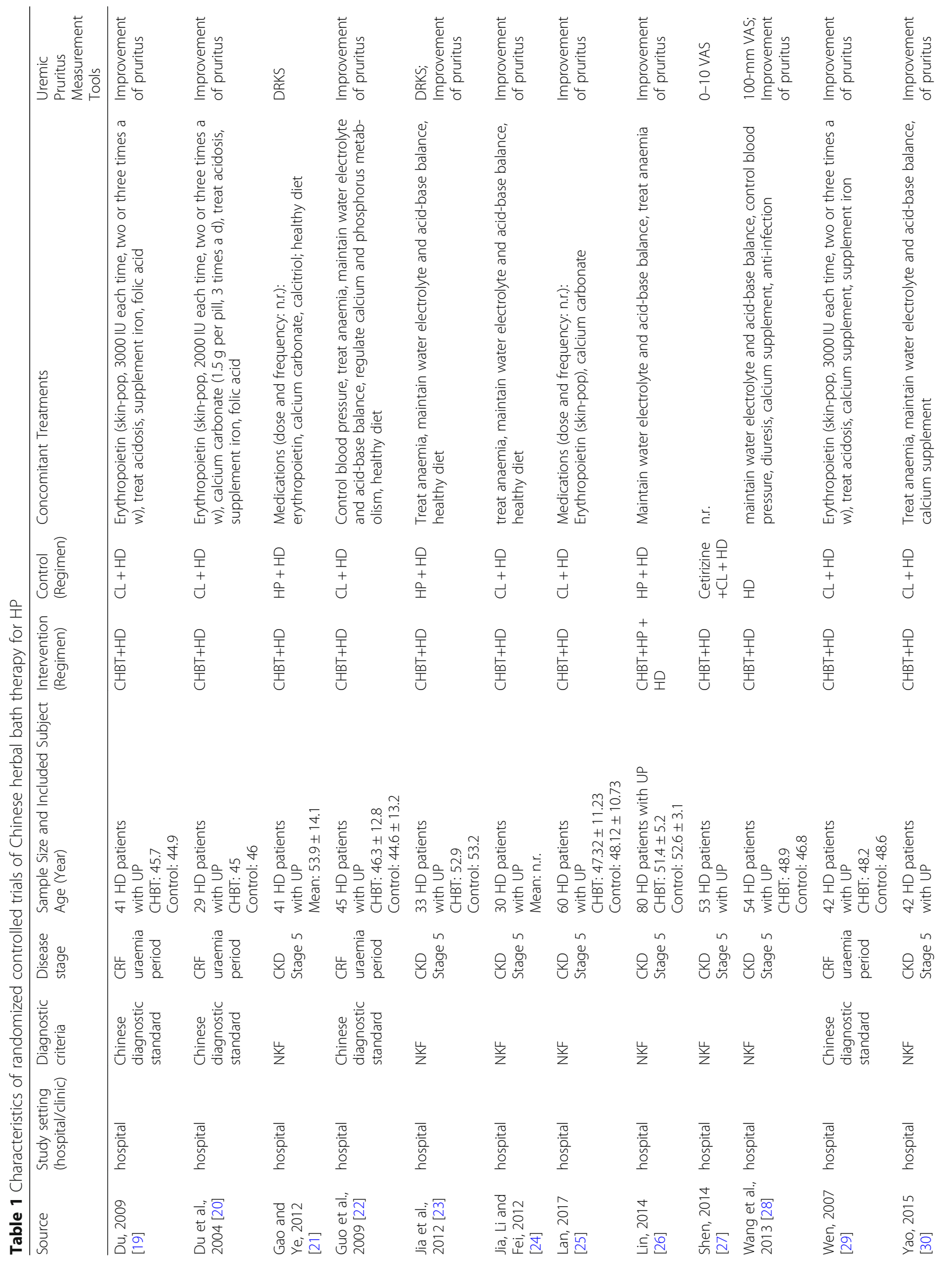




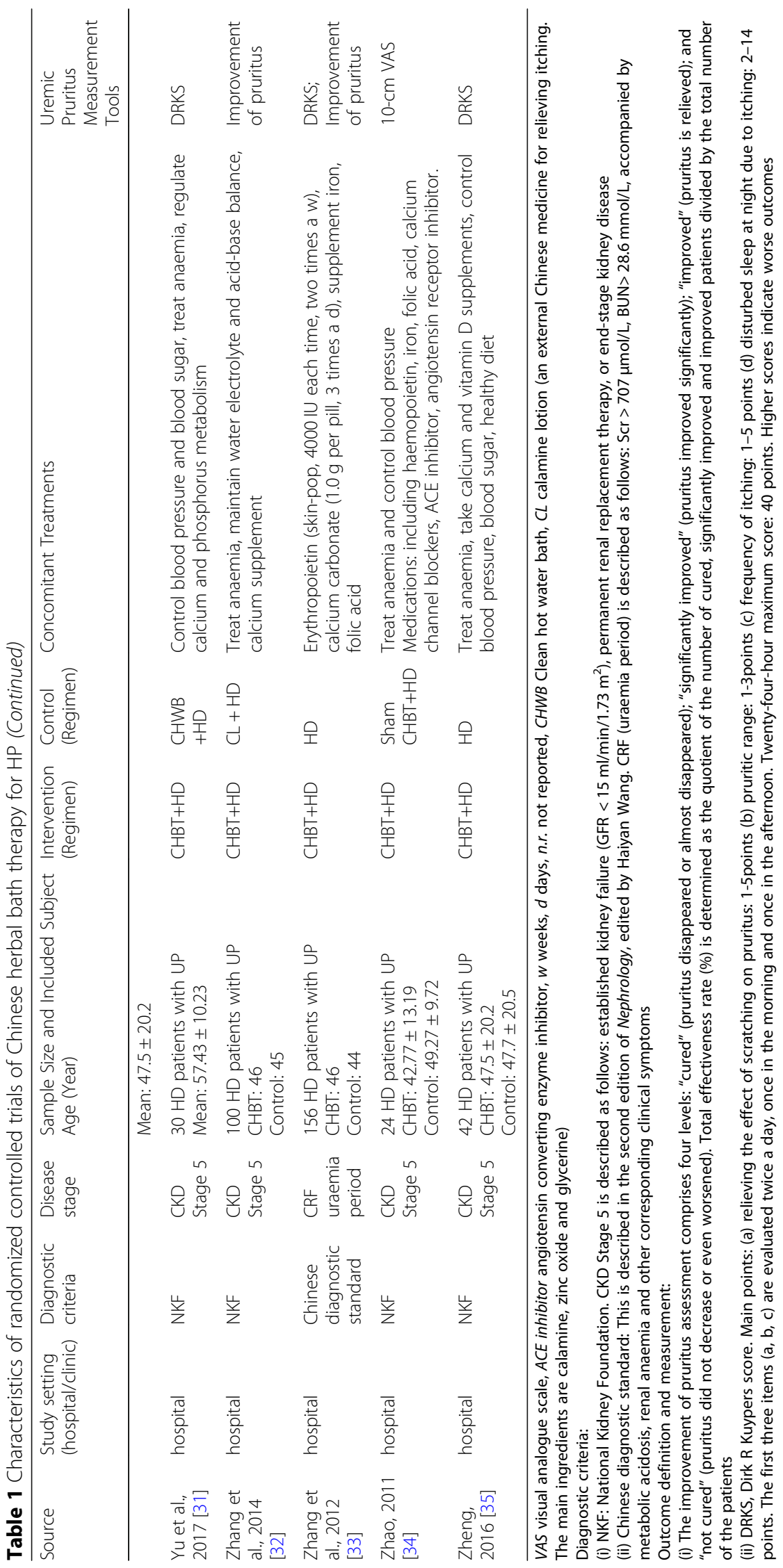


Table 2 Treatment details of included studies

\begin{tabular}{|c|c|c|c|c|c|}
\hline \multirow[t]{2}{*}{ Study } & \multicolumn{2}{|c|}{ CHBT group intervention } & \multicolumn{2}{|c|}{ Control group intervention } & \multirow[t]{2}{*}{ Duration } \\
\hline & $\begin{array}{l}\text { frequency of HD } \\
\text { or HP }\end{array}$ & $\mathrm{CHBT}$ & $\begin{array}{l}\text { frequency of } \mathrm{HD} \\
\text { or HP }\end{array}$ & other methods & \\
\hline $\begin{array}{l}\text { Du, } 2009 \\
{[19]}\end{array}$ & $\begin{array}{l}\text { HD: } 2 \text { or } 3 \text { times a } \\
\text { w, } 4 \mathrm{~h} \text { each time }\end{array}$ & $\begin{array}{l}10 \text { herbs: } 30-100 \mathrm{~g} \text { each, } \\
\text { bathed for } 30-40 \text { min, once a d }\end{array}$ & $\begin{array}{l}\text { HD: same as the } \\
\text { CHBT group }\end{array}$ & $C L$ : external use, three times a d & $30 \mathrm{~d}$ \\
\hline $\begin{array}{l}\text { Du et al., } \\
2004[20]\end{array}$ & $\begin{array}{l}\text { HD: } 2 \text { or } 3 \text { times a } \\
\text { w, } 4 \text { to } 5 \mathrm{~h} \text { each } \\
\text { time }\end{array}$ & $\begin{array}{l}9 \text { herbs: } 30-150 \mathrm{~g} \text { each, } \\
\text { bathed for } 30-60 \mathrm{~min} \text {, } \\
\text { once every two d }\end{array}$ & $\begin{array}{l}\text { HD: same as the } \\
\text { CHBT group }\end{array}$ & $C L$ : external use, 3 times a d & $30 \mathrm{~d}$ \\
\hline $\begin{array}{l}\text { Gao and } \\
\text { Ye, } 2012 \\
{[21]}\end{array}$ & HD: 9 to $12 \mathrm{~h}$ a w & $\begin{array}{l}10 \text { herbs: } 15-50 \mathrm{~g} \text { each; } \\
\text { WT: } 40-45^{\circ} \mathrm{C} \text {; bathed for } 20-30 \mathrm{~min} \text {; } \\
\text { three times a w }\end{array}$ & $\begin{array}{l}\text { HD: same as the } \\
\text { CHBT group } \\
\text { HP: two h each } \\
\text { time, once every } \\
\text { two w }\end{array}$ & n.r. & $3 \mathrm{~m}$ \\
\hline $\begin{array}{l}\text { Guo et al., } \\
2009 \text { [22] }\end{array}$ & $\begin{array}{l}\text { HD: once every } 2 \\
\text { to } 3 \mathrm{~d}, 4 \mathrm{~h} \text { each } \\
\text { time }\end{array}$ & $\begin{array}{l}10 \text { herbs: } 20-50 \mathrm{~g} \text { each, } \mathrm{WT}: 40-45^{\circ} \mathrm{C} \text {; } \\
\text { bathed for } \\
30-60 \text { min, once a d }\end{array}$ & $\begin{array}{l}\text { HD: same as the } \\
\text { CHBT group }\end{array}$ & $\begin{array}{l}\text { CL: external use, two or three times } \\
\text { a d }\end{array}$ & $2 w$ \\
\hline $\begin{array}{l}\text { Jia et al., } \\
2012[23]\end{array}$ & $\begin{array}{l}\text { HD: } 2 \text { or } 3 \text { times a } \\
\text { w; } \\
\text { HP: once every } \\
\text { two w, } 2 \text { h each } \\
\text { time }\end{array}$ & $\begin{array}{l}11 \text { herbs: } 15-50 \mathrm{~g} \text { each; } \mathrm{WT}: 40-43^{\circ} \mathrm{C} \text {; } \\
\text { bathed for } 30 \text { min; once a d }\end{array}$ & $\begin{array}{l}\text { HD, HP: same as } \\
\text { the CHBT group }\end{array}$ & n.r. & $8 w$ \\
\hline $\begin{array}{l}\text { Jia, Li and } \\
\text { Fei, } 2012 \\
{[24]}\end{array}$ & HD: regular. & $\begin{array}{l}11 \text { herbs: } 15-50 \mathrm{~g} \text { each; } \\
\text { WT: } 40-43^{\circ} \mathrm{C} \text {; bathed for } 30 \mathrm{~min} \text {; } \\
\text { once a d }\end{array}$ & $\begin{array}{l}\text { HD: same as the } \\
\text { CHBT group }\end{array}$ & external use, three or four times a $d$ & $4 w$ \\
\hline $\begin{array}{l}\text { Lan, } 2017 \\
{[25]}\end{array}$ & $\begin{array}{l}\text { HD: } 2 \text { or } 3 \text { times a } \\
w, 4 h \text { each time }\end{array}$ & $\begin{array}{l}10 \text { herbs: } 20-50 \mathrm{~g} \text { each, bathed for } 30-60 \mathrm{~min} \text {, } \\
\text { once every two d }\end{array}$ & $\begin{array}{l}\text { HD: same as the } \\
\text { CHBT group }\end{array}$ & $C L$ : external use, 2 or 3 times a $d$ & $30 \mathrm{~d}$ \\
\hline $\begin{array}{l}\operatorname{Lin}, 2014 \\
{[26]}\end{array}$ & $\begin{array}{l}\text { HD: } 2 \text { or } 3 \text { times a } \\
\text { W; } \\
\text { HP: } 2 \text { times a } m\end{array}$ & $\begin{array}{l}11 \text { herbs: } 15-50 \mathrm{~g} \text { each, } \mathrm{WT} \text { : approximately } \\
42^{\circ} \mathrm{C} \text {, bathed for } 30 \mathrm{~min} \text {, once a d }\end{array}$ & $\begin{array}{l}\text { HD: } 2 \text { or } 3 \text { times a } \\
\text { W; } \\
\text { HP: } 2 \text { times a } m\end{array}$ & n.r. & $2 m$ \\
\hline $\begin{array}{l}\text { Shen, } 2014 \\
{[27]}\end{array}$ & HD: regular. & 14 herbs, bathed for 30-40 min, once a d & $\begin{array}{l}\text { HD: same as the } \\
\text { CHBT group }\end{array}$ & $\begin{array}{l}\mathrm{CL} \text { : external use } \\
\text { Cetirizine: take orally } \\
\text { Dose and frequency: n.r. }\end{array}$ & $2 w$ \\
\hline $\begin{array}{l}\text { Wang et } \\
\text { al., } 2013 \\
{[28]}\end{array}$ & HD: regular. & $\begin{array}{l}11 \text { herbs: } 5-30 \mathrm{~g} \text { each, bathed for } 30 \mathrm{~min} \text {, } \\
\text { once a d }\end{array}$ & $\begin{array}{l}\text { HD: same as the } \\
\text { CHBT group }\end{array}$ & n.r. & $4 w$ \\
\hline $\begin{array}{l}\text { Wen, } 2007 \\
{[29]}\end{array}$ & $\begin{array}{l}\text { HD: } 2 \text { or } 3 \text { times a } \\
\text { w, } 4 \text { to } 5 \mathrm{~h} \text { each } \\
\text { time }\end{array}$ & $\begin{array}{l}9 \text { herbs: } 30-200 \mathrm{~g} \text { each, bathed for } 30-60 \mathrm{~min} \text {, } \\
\text { once every two d }\end{array}$ & $\begin{array}{l}\text { HD: same as the } \\
\text { CHBT group }\end{array}$ & CL: external use, 3 times a d & $20 d$ \\
\hline $\begin{array}{l}\text { Yao, } 2015 \\
{[30]}\end{array}$ & HD: regular. & $\begin{array}{l}6 \text { kinds of Chinese medicine granules: } 10-30 \\
\text { g each, WT: } 40-45^{\circ} \mathrm{C} \text {, bathed for } 15-30 \mathrm{~min} \text {, } \\
\text { once a d }\end{array}$ & $\begin{array}{l}\text { HD: same as the } \\
\text { CHBT group }\end{array}$ & $\begin{array}{l}\text { CL: external use, two or three times } \\
\text { a d }\end{array}$ & $2 w$ \\
\hline $\begin{array}{l}\text { Yu et } \\
\text { al.,2017 } \\
{[31]}\end{array}$ & $\begin{array}{l}\text { HD: } 3 \text { times a } w, 4 \\
\text { h each time }\end{array}$ & $\begin{array}{l}10 \text { herbs: } 50-100 \mathrm{~g} \text { each, hot water, bathed } \\
\text { for } 30 \text { min, once a d }\end{array}$ & $\begin{array}{l}\text { HD: same as the } \\
\text { CHBT group }\end{array}$ & $\begin{array}{l}\text { Clear hot water bath: } 30 \mathrm{~min} \text { at a } \\
\text { time }\end{array}$ & $2 w$ \\
\hline $\begin{array}{l}\text { Zhang et } \\
\text { al., } 2014 \\
{[32]}\end{array}$ & HD: regular. & $\begin{array}{l}6 \text { kinds of Chinese medicine granules, WT: } \\
40-45^{\circ} \mathrm{C} \text {, bathed for } 15-30 \mathrm{~min} \text {, once a d }\end{array}$ & $\begin{array}{l}\text { HD: same as the } \\
\text { CHBT group }\end{array}$ & $\begin{array}{l}\text { CL: external use, two or three times } \\
\text { a d }\end{array}$ & $2 w$ \\
\hline $\begin{array}{l}\text { Zhang et } \\
\text { al., } 2012 \\
{[33]}\end{array}$ & HD: regular. & 13 herbs, warm water, once a d & $\begin{array}{l}\text { HD: same as the } \\
\text { CHBT group }\end{array}$ & n.r. & $2 w$ \\
\hline $\begin{array}{l}\text { Zhao, } 2011 \\
{[34]}\end{array}$ & HD: regular. & $\begin{array}{l}22 \text { herbs, WT: } 37-40^{\circ} \mathrm{C} \text {, bathed for } 30-40 \mathrm{~min} \text {, } \\
\text { once a d }\end{array}$ & $\begin{array}{l}\text { HD: same as the } \\
\text { CHBT group }\end{array}$ & $\begin{array}{l}\text { Sham CHBT: water with the same } \\
\text { colour and smell as the } \\
\text { experimental group }\end{array}$ & $2 w$ \\
\hline $\begin{array}{l}\text { Zheng, } \\
2016 \text { [35] }\end{array}$ & $\begin{array}{l}\text { HD: } 2 \text { or } 3 \text { times a } \\
w, 4 h \text { each time }\end{array}$ & 9 herbs, bathed for 30 min, once a d & $\begin{array}{l}\text { HD: same as the } \\
\text { CHBT group }\end{array}$ & n.r. & $3 \mathrm{~m}$ \\
\hline
\end{tabular}

WT water temperature, $m$ months, $w$ weeks, $d$ days, $h$ hours, n.r. not reported 


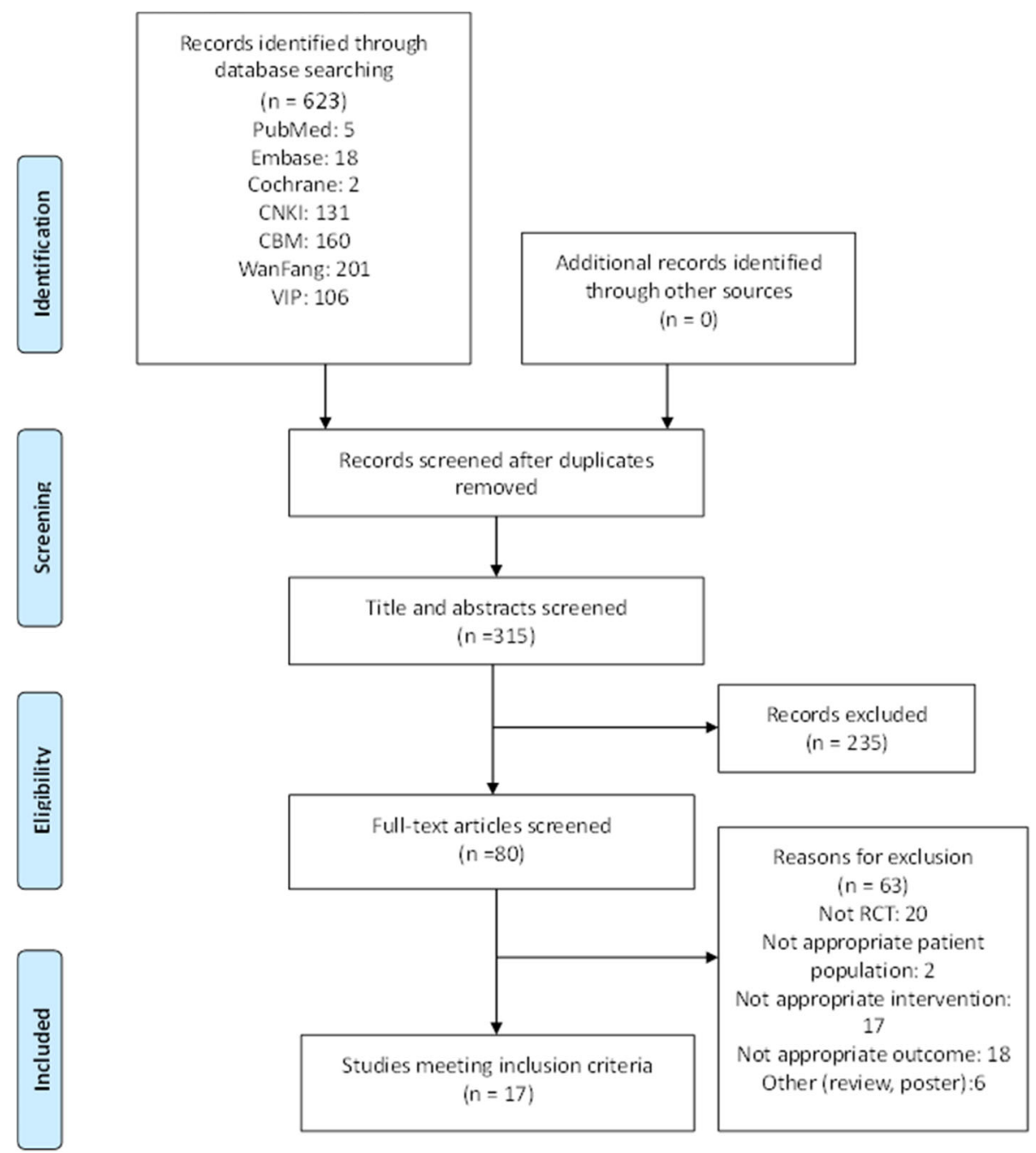

Fig. 1 Study selection flow chart

\section{Continuous data outcomes}

We unified the VAS of the three trials involving 111 patients into VAS (0-10 points) and conducted statistical analysis according to the data after conversion. The random-effects model was used. Subgroup analysis was performed because of clinical heterogeneity between the different control groups. The comprehensive analysis showed that the pruritus scores of patients in the Chinese herbal bath therapy combined with those of the haemodialysis groups were significantly lower than those in the control groups $(\mathrm{MD}=-2.38$; $95 \%$ confidence intervals $[\mathrm{CI}],-3.02$ to $-1.74 ; P<0.00001)$ after $2-4$ weeks of treatment. Subgroup results also support this conclusion. The second subgroup ( $\mathrm{CHBT}+\mathrm{HD}$ VS HD) showed that CHBT may indeed have an effect on the treatment of UP, whereas the other two groups showed better effectiveness than sham CHBT and cetirizine. There was no evidence for statistical heterogeneity across studies $($ chi-square $=0.49$; degree of freedom $=2$; $I^{2}=0 \%$ ) (Fig. 2).

Five trials involving 349 patients measured pruritus score based on the Dirk R Kuypers score (0-40 points). We used the random-effects model for statistical analysis. Subgroup analysis was performed according to the different controls. The pooled analysis showed that the pruritus scores of patients in the Chinese herbal bath therapy combined with the haemodialysis or haemoperfusion groups were lower than those in the control groups $(\mathrm{MD}=-8.42 ; 95 \%$ confidence intervals $[\mathrm{CI}]$, 12.47 to $-4.36 ; P<0.00001)$ after 2 weeks to 3 months of treatment. Three subgroup results also supported this conclusion and reflected the same situation. CHBT may be more effective than HP and a hot water bath. However, the combined analysis has significant statistical heterogeneity (chi-square $=196.02$; degree of freedom $=4$; $I^{2}=98 \%$ ) (Fig. 3). It was difficult to analyse the causes of 
Table 3 The 19 most frequently used flavours of Chinese herbal medicine in 17 bath prescriptions

\begin{tabular}{|c|c|c|c|}
\hline English name & Latin name & Chinese Pinyin name & Frequency of usage \\
\hline \multicolumn{4}{|l|}{ Pruritus relief } \\
\hline Fructus Kochiae & Fructus Kochiae Scopariae & Difuzi & 15 \\
\hline Lightyellow Sophora Root & Radix Sophorae Flavescentis & Kushen & 13 \\
\hline Densefruit Pittany Root-bark & Cortex Dictamni & Baixianpi & 13 \\
\hline Cicada Slough & Periostracum Cicadae & Chantui & 12 \\
\hline Common Cnidium Fruit & Fructus Cnidii & Shechuangzi & 4 \\
\hline Chinese Honeylocust Spine & Spina Gleditsiae & Zaojiaoci & 3 \\
\hline Puncturervine Caltrop Fruit & Fructus Tribuli Terrestris & Baijli & 3 \\
\hline Tuber Fleeceflower Root & Radix Polygoni Multiflori & Heshouwu & 3 \\
\hline \multicolumn{4}{|l|}{ Activating blood circulation } \\
\hline Chinese Angelica & Radix Angelicae Sinensis & Danggui & 12 \\
\hline Manchurian Wildginger & Herba Asari & Xixin & 12 \\
\hline Szechuan Lovage Rhizome & Rhizoma Chuanxiong & Chuanxiong & 11 \\
\hline Suberect Spatholobus Stem & Caulis Spatholobi & Jixueteng & 5 \\
\hline Dan-shen root & Radix Salviae Miltiorrhizae & Danshen & 4 \\
\hline \multicolumn{4}{|l|}{ Stimulating secretion of sweat } \\
\hline Chinese Ephedrs Herb & Ephedra sinica Stapf & Mahuang & 7 \\
\hline Fineleaf Schizonepeta Herb & Herba Schizonepetae & Jingjie & 8 \\
\hline Angelica Dahurica & Radix Angelicae Dahuricae & Baizhi & 6 \\
\hline Divaricate Saposhniovia Root & Radix Saposhnikoviae & Fangfeng & 4 \\
\hline \multicolumn{4}{|l|}{ Promoting detoxification } \\
\hline Rhubarb Root and Rhizome & Rheum Officinale Baill & Dahuang & 7 \\
\hline
\end{tabular}

\begin{tabular}{|c|c|c|c|c|c|c|c|c|c|c|c|}
\hline \multirow[b]{2}{*}{ Study or Subgroup } & \multicolumn{3}{|c|}{ Experimental } & \multicolumn{2}{|c|}{ Control } & \multirow{2}{*}{\multicolumn{2}{|c|}{ Total Weight }} & \multirow{2}{*}{$\begin{array}{l}\text { Mean Difference } \\
\text { IV. Random, } 95 \% \mathrm{CI}\end{array}$} & \multirow{2}{*}{\multicolumn{3}{|c|}{$\begin{array}{c}\text { Mean Difference } \\
\text { IV. Random, } 95 \% \mathrm{Cl}\end{array}$}} \\
\hline & Mean & & Total & Mean & & & & & & & \\
\hline \multicolumn{12}{|c|}{ 1.1.1 CHBT+HD VS CL+HD+Cetirizine } \\
\hline $\begin{array}{l}\text { Shen, } 2014 \\
\text { Subtotal }(95 \% \mathrm{CI})\end{array}$ & -4.98 & 1.12 & $\begin{array}{l}26 \\
26\end{array}$ & -2.38 & 2.42 & $\begin{array}{l}27 \\
27\end{array}$ & $\begin{array}{l}40.1 \% \\
40.1 \%\end{array}$ & $\begin{array}{l}-2.60[-3.61,-1.59] \\
-2.60[-3.61,-1.59]\end{array}$ & 1 & & \\
\hline \multicolumn{12}{|c|}{ Heterogeneity: Not applicable } \\
\hline \multicolumn{12}{|c|}{ 1.1.2 CHBT+HD VS HD } \\
\hline $\begin{array}{l}\text { Wang et al., } 2013 \\
\text { Subtotal }(95 \% \mathrm{Cl})\end{array}$ & -3.737 & 1.636 & $\begin{array}{l}17 \\
17\end{array}$ & -1.333 & 1.662 & $\begin{array}{l}17 \\
17\end{array}$ & $\begin{array}{l}33.3 \% \\
33.3 \%\end{array}$ & $\begin{array}{l}-2.40[-3.51,-1.30] \\
-2.40[-3.51,-1.30]\end{array}$ & 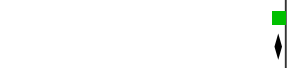 & & \\
\hline \multicolumn{11}{|c|}{ Heterogeneity: Not applicable } & Test for overall effect: $Z=4.25(P<0.0001)$ \\
\hline \multicolumn{12}{|c|}{ 1.1.3 CHBT+HD VS Sham CHBT+HD } \\
\hline $\begin{array}{l}\text { Yu et al.,2017 } \\
\text { Subtotal }(95 \% \mathrm{Cl})\end{array}$ & -2.72 & 1.4 & $\begin{array}{l}13 \\
13\end{array}$ & -0.69 & 1.655 & $\begin{array}{l}11 \\
11\end{array}$ & $\begin{array}{l}26.6 \% \\
26.6 \%\end{array}$ & $\begin{array}{l}-2.03[-3.27,-0.79] \\
-2.03[-3.27,-0.79]\end{array}$ & 1 & & \\
\hline \multicolumn{12}{|c|}{$\begin{array}{l}\text { Heterogeneity: Not applicable } \\
\text { Test for overall effect: } Z=3.21(P=0.001)\end{array}$} \\
\hline Total $(95 \% \mathrm{Cl})$ & & & 56 & & & 55 & $100.0 \%$ & $-2.38[-3.02,-1.74]$ & 1 & & \\
\hline \multicolumn{9}{|c|}{$\begin{array}{l}\text { Heterogeneity: } \mathrm{Tau}^{2}=0.00 ; \mathrm{Chi}^{2}=0.49, \mathrm{df}=2(P=0.78) ; \mathrm{I}^{2}=0 \% \\
\text { Test for overall effect: } Z=7.31(P<0.00001) \\
\text { Test for subgroup differences: } \mathrm{Chi}^{2}=0.49, \mathrm{df}=2(\mathrm{P}=0.78), \mathrm{I}^{2}=0 \%\end{array}$} & $\begin{array}{ccc}-100 & -50 & 0 \\
\text { Favours [experimental] }\end{array}$ & $\begin{array}{c}50 \\
\text { Favours [control] }\end{array}$ & 100 \\
\hline Fig. 2 Effect of Chine & e herbal & bath th & lerapy & on pru & uritus sc & core $(\mathrm{V}, \mathrm{r}$ & & & & & \\
\hline
\end{tabular}




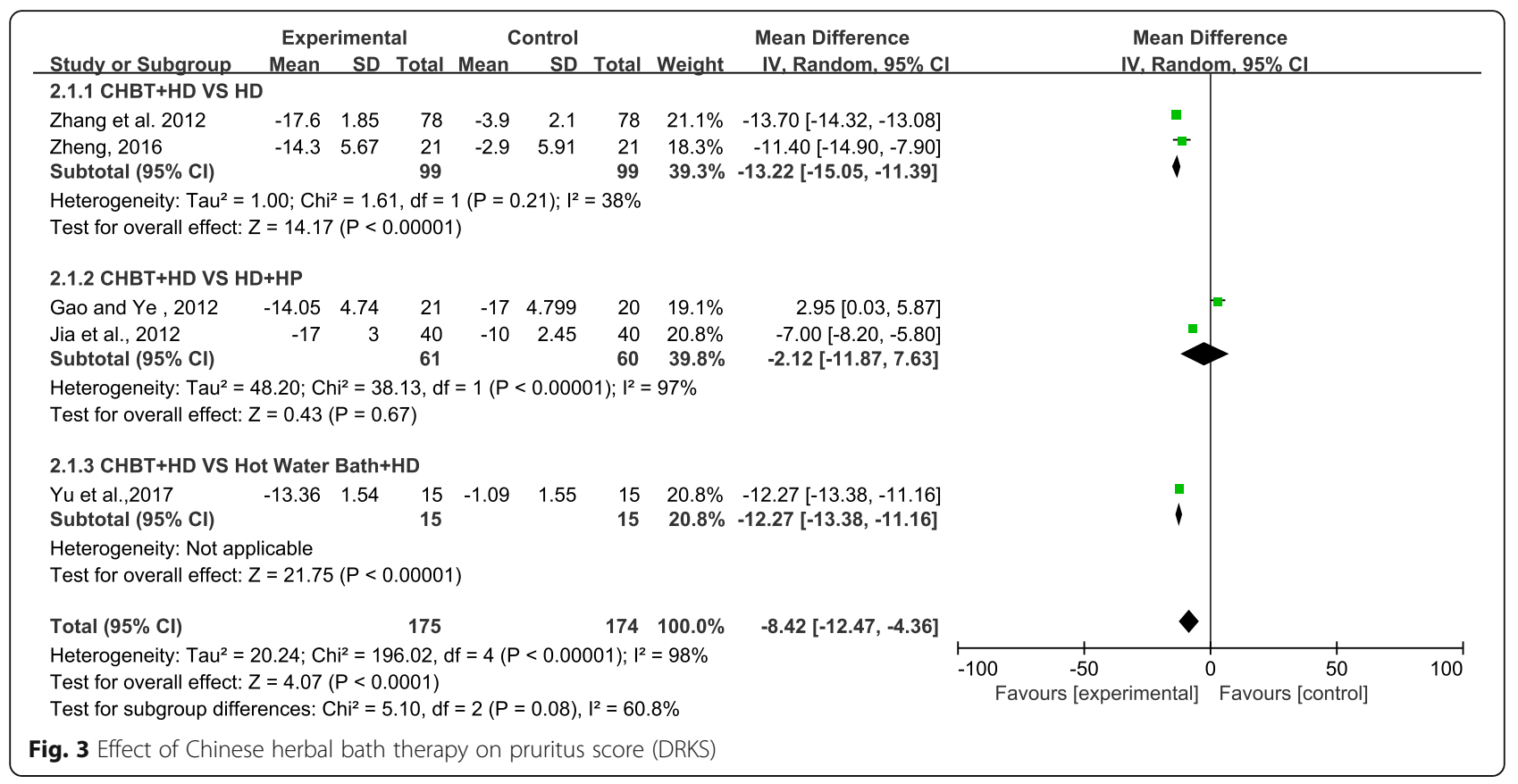

heterogeneity due to the small number of studies. The reason maybe that the index value span ( $0-40$ points) is too large, and the scoring process is subjective according to the patients.

As mentioned above, the pruritic score of the patients in Chinese herbal bath therapy was lower than that of the control groups.

\section{Total effectiveness rate outcomes}

Twelve trials involving 780 patients reported a comparison of the total effectiveness rate between Chinese herbal bath therapy combined with modern basic medical treatment and control groups using modern basic medical treatment or adding calamine lotion. The results from our random-effects model meta-analysis revealed that Chinese herbal bath

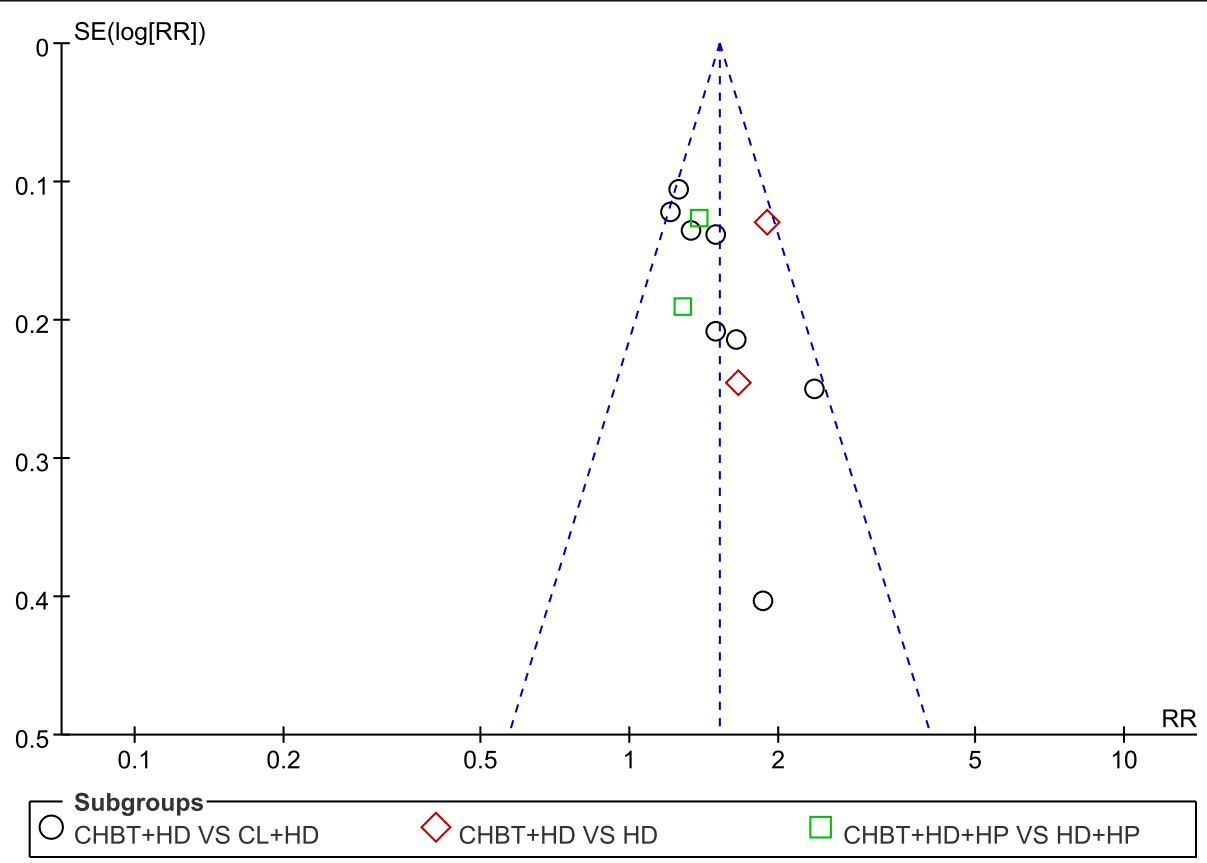

Fig. 4 Funnel plot of Chinese herbal bath therapy on overall effectiveness 
therapy combined with modern basic medical treatment improved the clinical effective rates by $46 \%$ on average when compared with controls (risk ratio $[\mathrm{RR}]=1.46 ; 95 \% \mathrm{CI}, 1.31$ to $1.63 ; P<0.00001$ ) after 2 weeks to 2 months of treatment, without significant heterogeneity among studies $\left(\mathrm{I}^{2}=27 \%\right)$. An asymmetrical funnel plot of the 12 trials presented potential publication bias as shown in Fig. 4.

We performed a further subgroup meta-analysis exploring the improvement of different controls on total effectiveness rate. The results showed that the combined use of Chinese herbal bath therapy and modern basic medical treatment has a better effect when compared with HD (RR $=1.84 ; 95 \% \mathrm{CI}, 1.47$ to $2.30 ; P<0.00001)$, HD combined with HP $(\mathrm{RR}=1.35$; $95 \% \mathrm{CI}, 1.11$ to $1.66 ; P<0.001)$ or calamine lotion $(\mathrm{RR}=1.41 ; 95 \% \mathrm{CI}, 1.24$ to $1.61 ; P<$ 0.00001) (Fig. 5). As described above, the RR values of subgroups $(\mathrm{CHBT}+\mathrm{HD}$ VS HD, $\mathrm{CHBT}+\mathrm{HD}+\mathrm{HP}$ VS HD $+\mathrm{HP}$ ) greater than 1 indicated that CHBT may be effective in treating UP, whereas the RR value of the group $(\mathrm{CHBT}+\mathrm{HD}$ VS $\mathrm{CL}+\mathrm{HD})$ greater than 1 indicated that the CHBT may be more effective than calamine lotion.
Because different dosages of CHBT may lead to different therapeutic effects, we further analysed 8 RCTs in the first subgroup (CHBT+HD VS CL + HD).

First, we performed further subgroup analysis according to the different duration of each bath. The results of the subgroups were ranked in order of $R R$ value from large to small: each CHBT lasts for more than $30 \mathrm{~min}(\mathrm{RR}=1.59 ; 95 \% \mathrm{CI}, 1.23$ to $2.05 ; P<0.001)$, each CHBT lasts for $30 \mathrm{~min}(\mathrm{RR}=1.50$; $95 \% \mathrm{CI}, 1.14$ to 1.97; $P<0.01$ ), or each CHBT lasts from 15 to $30 \mathrm{~min}$ (RR =1.27; 95\% CI, 1.06 to 1.51; $P<0.01$ ) (Fig. 6). The results suggested that appropriate prolonging of each CHBT may be beneficial for the treatment of UP.

Second, we conducted subgroup analysis according to different bath frequency. The results of the two groups were ranked in order of $R R$ value from large to small: once a day $(\mathrm{RR}=1.47 ; 95 \% \mathrm{CI}, 1.22$ to 1.77 ; $P<0.001)$ or once every 2 days $(\mathrm{RR}=1.33 ; 95 \% \mathrm{CI}$, 1.11 to $1.60 ; P<0.01$ ) (Fig. 7 ). The results suggested that an herbal bath once a day may be more effective than an herbal bath once every other day.

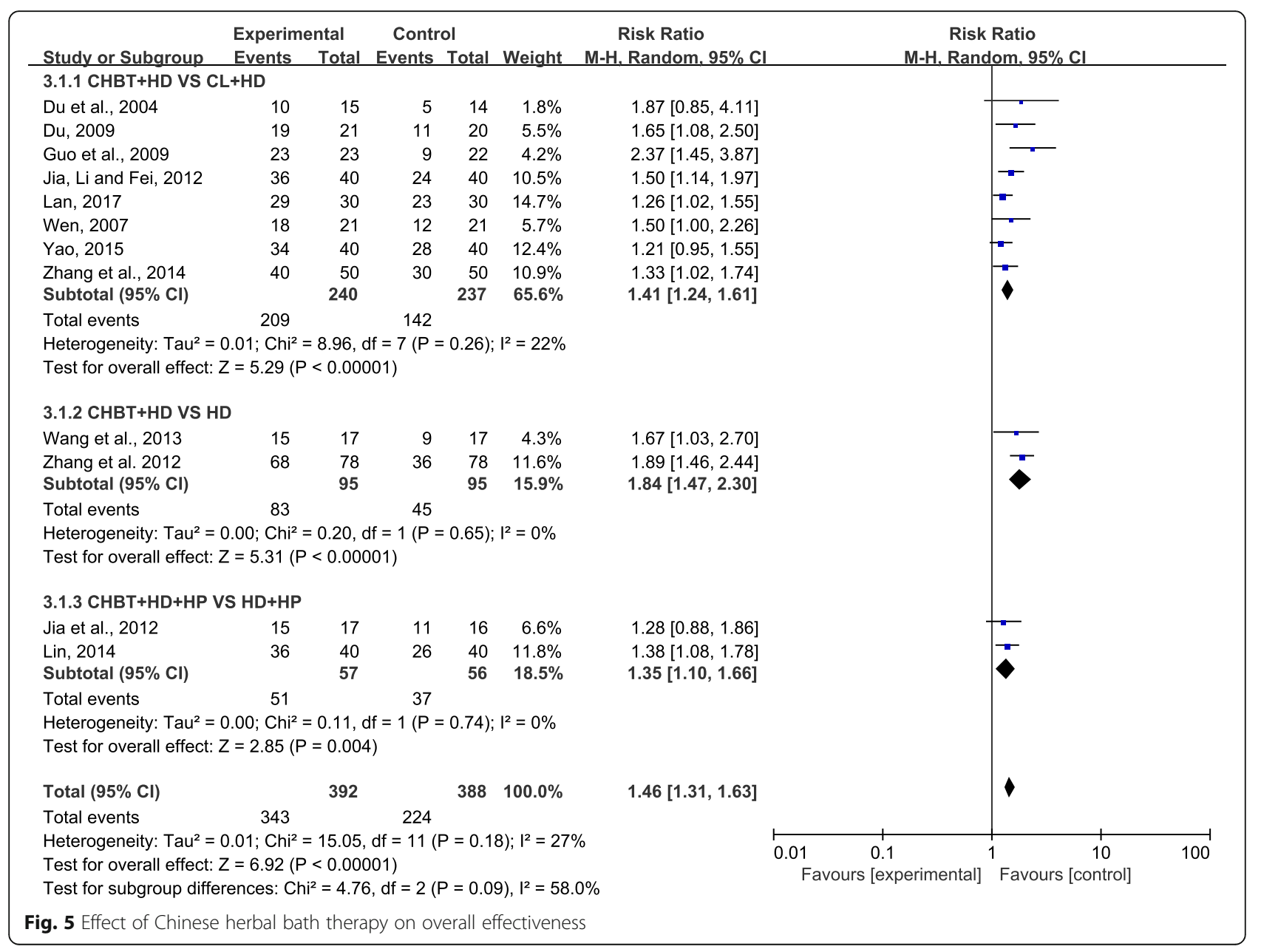




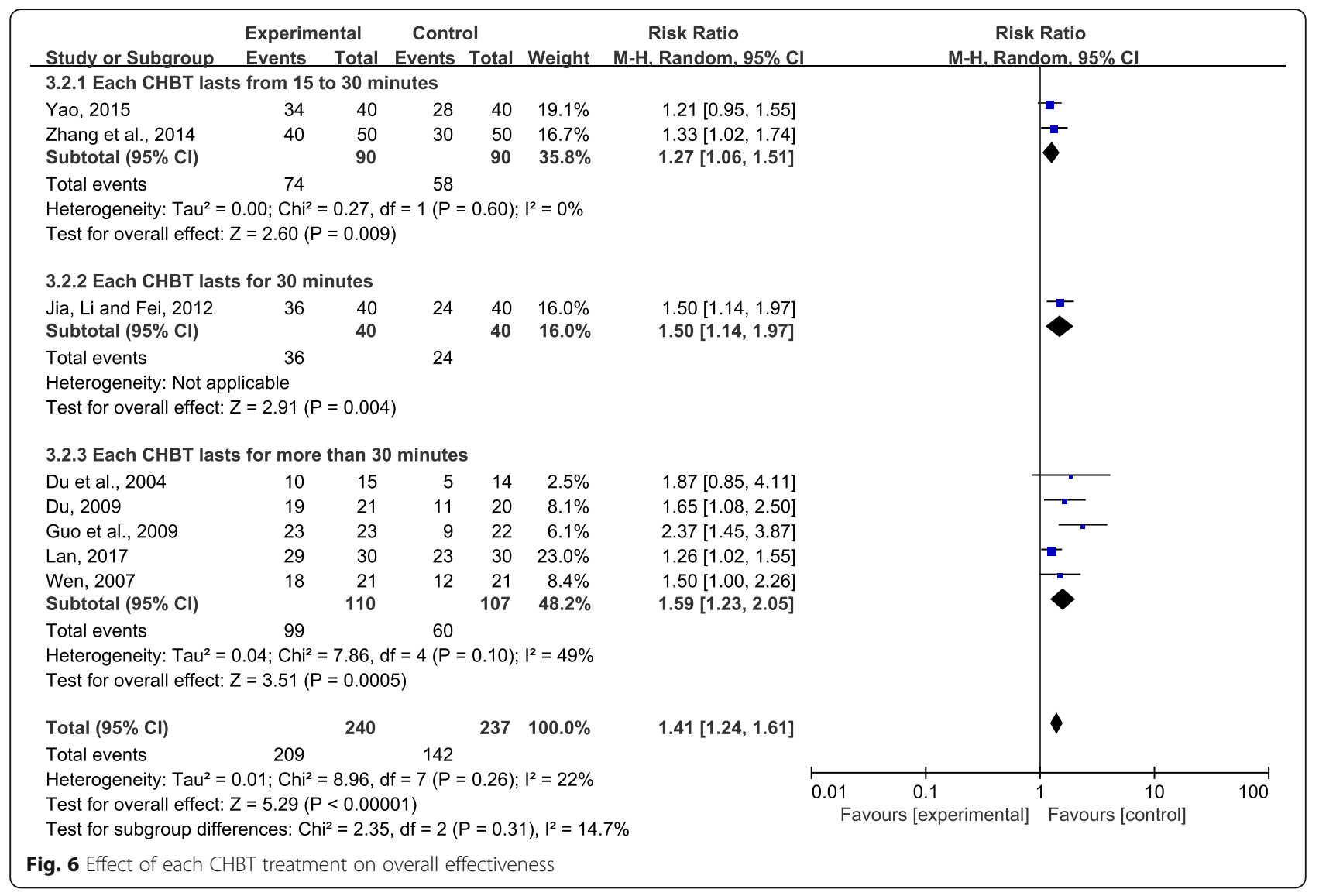

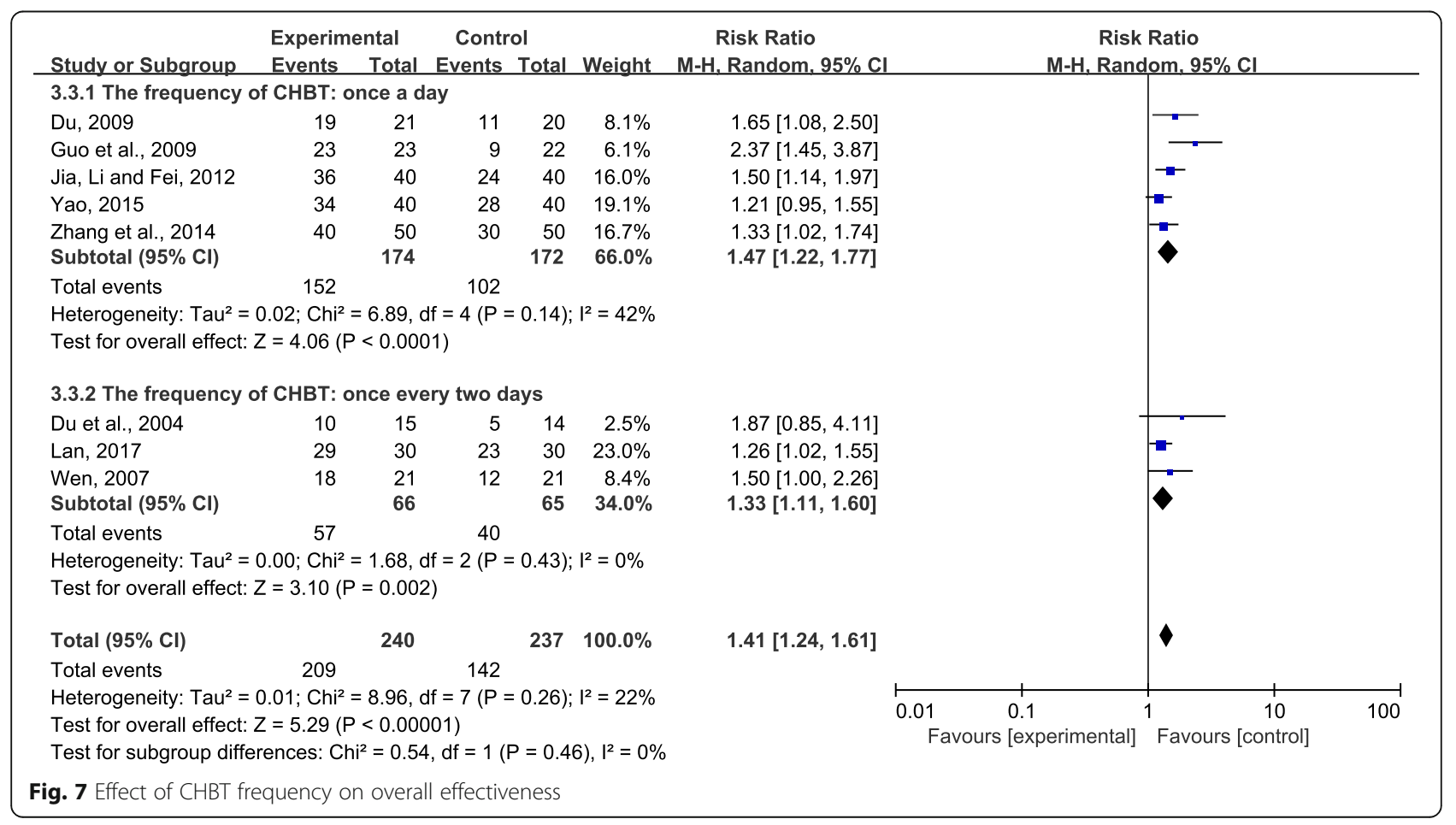


Third, we conducted a subgroup analysis based on the length of treatment duration. Due to the large $P$ values $(P \geq 0.05)$, the second subgroup (the treatment duration was 20 days) was not statistically significant. The results of the other two subgroups were ranked in order of RR value from large to small: treatment duration was 2 weeks $(\mathrm{RR}=1.47 ; 95 \% \mathrm{CI}, 1.07$ to $2.01 ; P<0.05)$ or treatment duration was 4 weeks or 1 month $(\mathrm{RR}=$ 1.40; 95\% CI, 1.20 to $1.62 ; P<0.0001$ ) (Fig. 8). The results showed that the short-term effect of herbal bath may be more obvious than the long-term effect in the treatment of UP.

Overall, compared with basic treatment (HD or HP) and adding combination with sham CHBT, clean hot water bath, or calamine lotion, all RCTs reported that Chinese herbal bath therapy plus basic treatment significantly relieved itching after 2 weeks to 3 months of treatment. However, the therapeutic effects of different herbal bath dosages is not very clear, and the long-term effect of CHBT for UP needs further study.

\section{Adverse events}

Seven RCTs suggested that patients stop bathing if they felt unwell during the herbal bath treatment, but no adverse events were mentioned in any of the included RCTs. In addition, for safety reasons, 8 RCTs recommended adjusting the water temperature and bathing time according the patient's physical condition, and 4 RCTs deemed that patients should not bathe when they are particularly hungry and full.

\section{Methodological quality}

We assessed the risk of bias via the Cochrane Risk of Bias. According to the details of the results are presented in Fig. 9, the overall quality of the included trials was poor while two of them are moderate. All RCTs mentioned randomization, but only 1 article illustrated the specific grouping method using a random number table. None of the RCTs described allocation concealment or assessor blinding. In addition, only one trial used patient blinding. Meanwhile, a high risk of selective reporting could have existed in two trials.

\section{Discussion}

Chinese herbal bath therapy is a traditional medicine treatment that involves pouring the decoction or extract of Chinese medicine into warm water and then bathing. The therapeutic method is easy to master and can be done by skilled patients themselves. Moreover, the

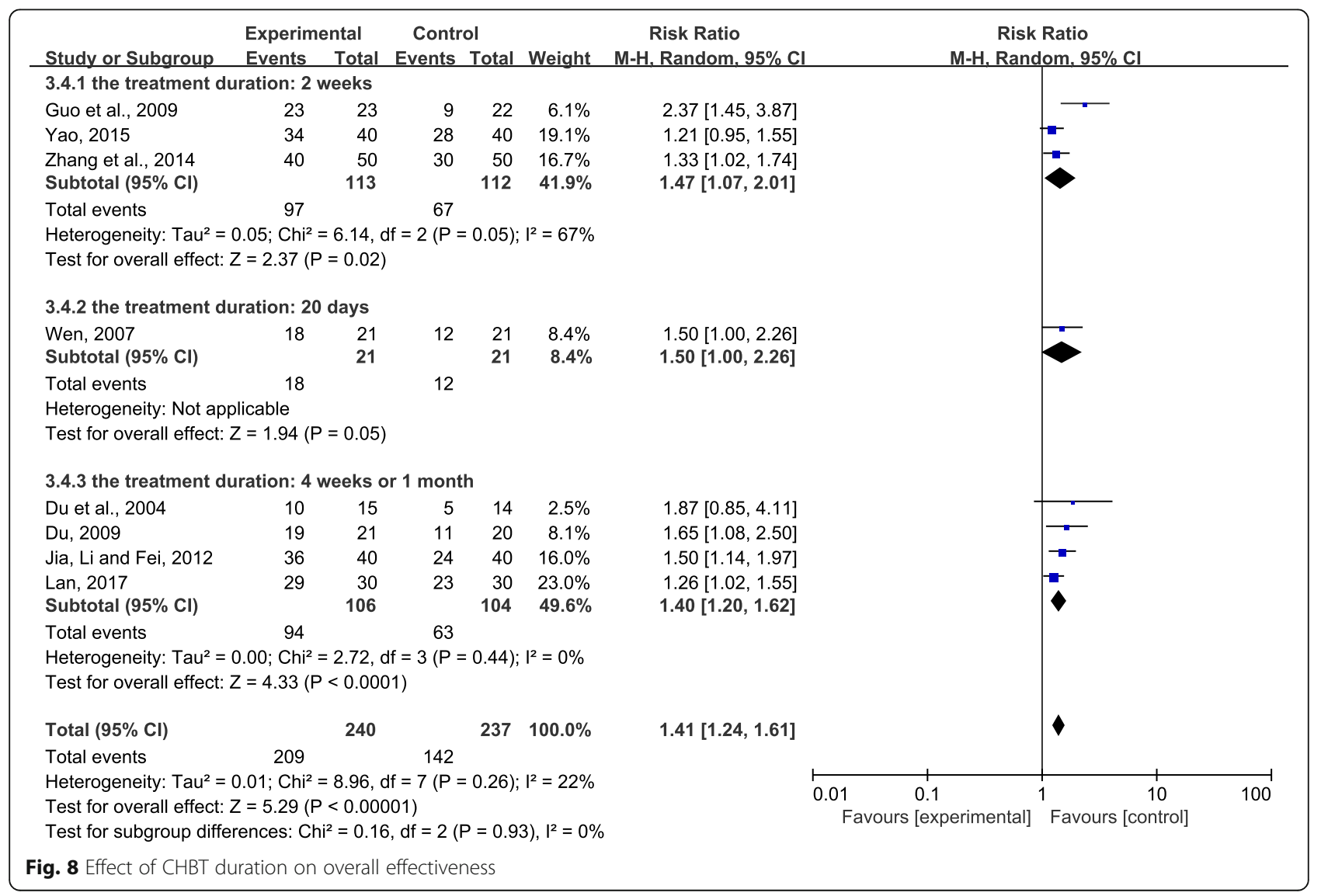




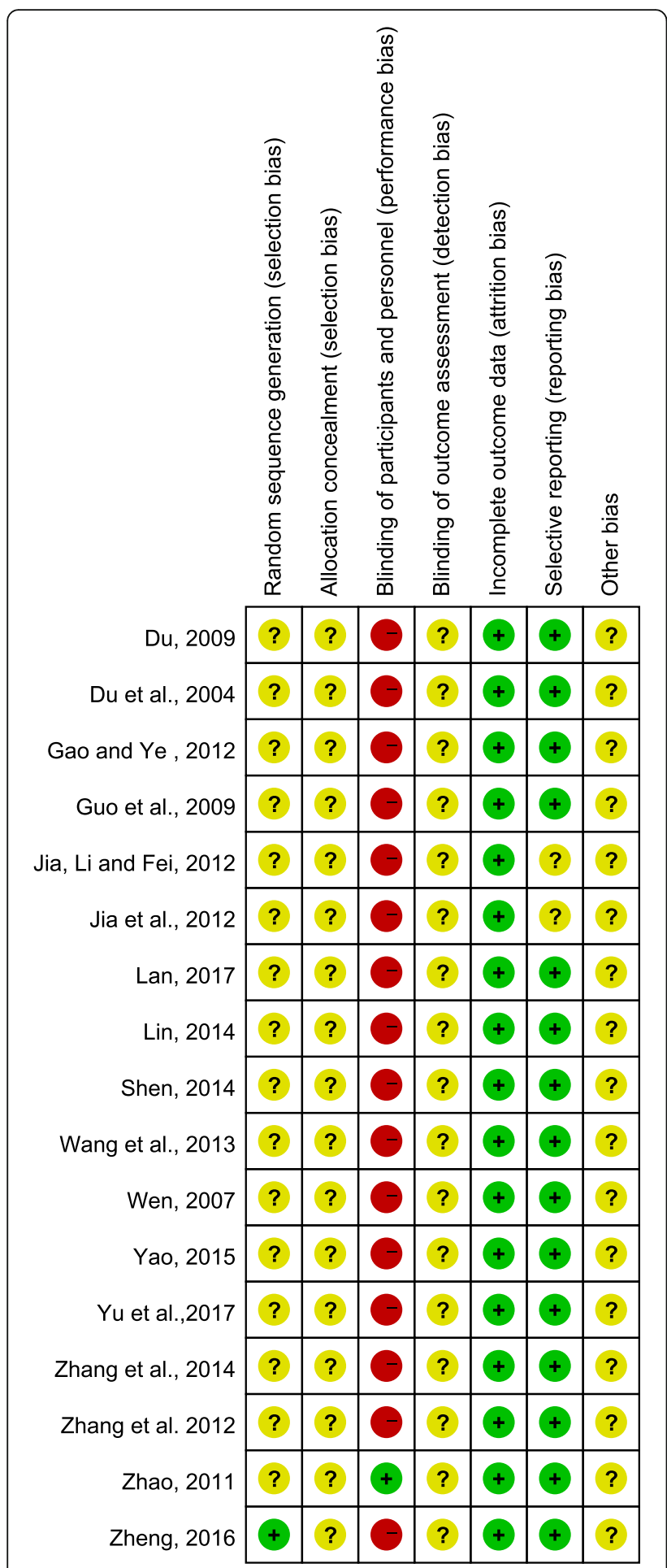

Fig. 9 Risk of bias in the included randomized controlled trials

herbal medicines used are not expensive and will not increase the financial burden of UP patients. In recent years, many clinical reports have shown that herbal bathing can effectively improve the symptoms of pruritus in patients with uraemia, and even assist in the treatment of uraemia [39-43]. This meta-analysis of 17 RCTs in 970 individuals also indicated that Chinese herbal bath therapy has a good adjuvant therapeutic effect on the treatment of UP. The results showed that itch relief of UP patients may be more significant with an appropriate increase in the frequency and duration of each bath. However, there is no evidence that the effect of herbal bath for UP is better in the long term than in the short term.

Despite the lack of interpretation about the biologic mechanisms of herbal bath therapy for uremic pruritus, the synergy between the efficacy of Chinese herbs and heating power may well be the basis for improving symptoms $[9,44]$. First, some Chinese medicated herbs have an anti-pruritic effect on the skin. The antipruritic herbs, such as Fructus Kochiae and Lightyellow Sophora Root, are commonly used in the herbal bath and can be absorbed through the skin [38, 45]. Second, some Chinese herbs described in Table 2 and hot water bath itself can accelerate blood circulation and promote metabolism. The hot stimulation of an herbal bath can dilate local blood vessels, promote local and peripheral blood circulation and lymphatic circulation, and facilitate the effective ingredients in the bath liquid to play a role in the whole body through blood circulation. Third, some Chinese herbs and hot water bath can promote sweating to eliminate metabolic toxins. Studies have shown that the concentration of urea in sweat fluid can reach 5 to 50 times that in the serum. Similarly, the creatinine levels in sweat fluid have been shown to be high in patients with urinuria [46-48]. Therefore, it is important to exclude urea and creatinine by sweating when kidney function is impaired. Song and Ma found that haemodialysis combined with herbal bath had a significant effect on the sweat composition of patients, which can increase the excretion of potassium, uric acid, urea nitrogen, phosphorus and creatinine, and reduce the output of $\beta 2$-microglobulin and parathyroid hormone in the sweat $[49,50]$. In addition, several RCTs [33, 35, 51-53] have revealed that herbal bath therapy can reduce the level of serum creatinine and blood urea nitrogen in patients with uraemia. With the elimination of toxins, itchy symptoms can be effectively relieved.

No adverse reactions were reported in the included RCTs. As an external treatment, water temperature and bath time can be controlled, so the safety of Chinese herbal bath therapy is high. However, in traditional Chinese medicine, the hot herbal bath was not used on extremely weak patients due further depletion of body fluids and some nutrients because of perspiration in a hot bath. Therefore, it is important to have a clear assessment of the physical condition of UP patients before the herbal bath treatment [54]. 
Overall, CHBT is effective, safe, simple and inexpensive. In view of the above advantages of herbal bath for UP, we believe that normative clinical guidelines should be developed in future. To achieve the goal, some difficult problems need to be solved. For example, different dosages of CHBT (frequency, time, duration) and different herbs used may lead to different therapeutic effects. Which dosages are the better choices? Which herbs are more effective? Which UP patients do not suit CHBT? These aspects need to be further studied.

There are several limitations in our study that should be noted. First, the overall methodological quality of the included trials was poor based on the Cochrane handbook. In fact, there were some methodological flaws that might involve a high risk of bias in most of the RCTs. Only one study [35] reported the details of randomization, and no trial mentioned double blinding. In addition, these RCTs generally lacked careful follow-up, and the treatment courses of some trials were only 2 weeks. Therefore, the long-term efficacy and safety of CHBT for UP were not easily assessed. Second, the publication bias, which was caused by all the studies being published in China, may exaggerate the efficacy of CHBT to some extent. Third, the included RCTs were all single-centre studies, and the sample size of the experimental studies was relatively small, which made the research results lack reliability. Fourth, there were some discrepancies in the interventions of the control groups in the trials. Consequently, the efficacy of Chinese drug bath therapy for UP needs to be further confirmed by more well-designed, large-scale clinical trials.

\section{Conclusion}

Chinese herbal bath therapy could be a complementary therapy to improve pruritic symptoms in uraemia patients. However, the methodological quality of these included trials was rated to be poor considering the risk of bias. More high-quality RCTs with rigorous designing are needed to further identify the efficacy and safety of CHBT for UP.

\section{Additional file}

Additional file 1: Links of the 17 included articles. (DOCX $21 \mathrm{~kb}$ )

\begin{abstract}
Abbreviations
CBM: Chinese Biomedical database; CHBT: Chinese herbal bath therapy; CL: Calamine lotion; CNKI: China Network Knowledge Infrastructure; DRKS: Dirk R Kuypers score; HD: Haemodialysis; HP: Haemoperfusion; MD: Mean difference; OTCMT: Qther traditional Chinese medicine treatment; PRISMA: Preferred Reporting Items for Systematic Review and Meta-analysis; RCT: Random Controlled Trial; RR: Risk ratio; SD: Standard deviation; UP: Uremic pruritus; VAS: Visual analogue scale; VIP: Chinese Scientific Journal Database
\end{abstract}

Acknowledgements

Not applicable.

\section{Funding}

This research received no specific grant from any funding agency in the public, commercial or not-for-profit sectors.

\section{Availability of data and materials}

We searched and analysed published articles to which anyone can gain access through different databases. In addition, the review protocol is available through PROSPERO (CRD42018108506).

\section{Authors' contributions}

WX and ZZ conceived and designed the study. WX and $Y Z$ performed the article search and data extraction. WX and MY evaluated the methodological quality of each study. WX analysed the data and wrote the paper, which was improved by $Y Z$ and MY. ZZ supervised the research. All authors read and approved the final manuscript.

\section{Ethics approval and consent to participate}

Not applicable.

\section{Consent for publication}

Not applicable.

\section{Competing interests}

The authors declare that they have no competing interests.

\section{Publisher's Note}

Springer Nature remains neutral with regard to jurisdictional claims in published maps and institutional affiliations.

Received: 4 December 2018 Accepted: 26 April 2019

Published online: 10 May 2019

\section{References}

1. Simonsen E, Komenda P, Lerner B, Askin N, Bohm C, Shaw J, Tangri N, Rigatto C. Treatment of uremic pruritus: a systematic review. Am J Kidney Dis. 2017;70(5):638-55.

2. Wikstrom B. Itchy skin--a clinical problem for haemodialysis patients. Nephrol Dial Transplant. 2007;22(Suppl 5):v3-7.

3. Combs SA, Teixeira JP, Germain MJ. Pruritus in kidney disease. Semin Nephrol. 2015:35(4):383-91.

4. Goodkin DA, Bieber B, Jadoul M, Martin P, Kanda E, Pisoni RL. Mortality, hospitalization, and quality of life among patients with hepatitis $C$ infection on hemodialysis. Clin J Am Soc Nephrol. 2017:12(2):287-97.

5. Mathur VS, Lindberg J, Germain M, Block G, Tumlin J, Smith M, Grewal M, McGuire D. A longitudinal study of uremic pruritus in hemodialysis patients. Clin J Am Soc Nephrol. 2010;5(8):1410-9.

6. Narita I, Alchi B, Omori K, Sato F, Ajiro J, Saga D, Kondo D, Skatsume M, Maruyama S, Kazama JJ, et al. Etiology and prognostic significance of severe uremic pruritus in chronic hemodialysis patients. Kidney Int. 2006;69(9): 1626-32.

7. Kimmel M, Alscher DM, Dunst R, Braun N, Machleidt C, Kiefer T, Stulten C, van der Kuip H, Pauli-Magnus $\mathrm{C}$, Raub $\mathrm{U}$, et al. The role of microinflammation in the pathogenesis of uraemic pruritus in haemodialysis patients. Nephrol Dial Transplant. 2006:21(3):749-55.

8. Chodorowska G, Wysokinski A, Chodorowski J. Uremic pruritus in the chronic renal failure patients. Ann Univ Mariae Curie-Sklodowska Med. 2004; 59(1):174-9.

9. Berger TG, Steinhoff M. Pruritus and renal failure. Semin Cutan Med Surg. 2011;30(2):99-100

10. Yong AS, Lee KY. Uremic pruritus is improved by gabapentin. Int J Dermatol. 2014;53(9):e404-5.

11. Makhlough A, Ala S, Haj-Heydari Z, Kashi Z, Bari A. Topical capsaicin therapy for uremic pruritus in patients on hemodialysis. Iran J Kidney Dis. 2010;4(2): 137-40.

12. Feily A, Dormanesh B, Ghorbani AR, Moosavi Z, Kouchak M, Cheraghian B, Mousavi SS, Mehrabian A, Ranjbari N. Efficacy of topical cromolyn sodium $4 \%$ on pruritus in uremic nephrogenic patients: a randomized double-blind study in 60 patients. Int J Clin Pharmacol Ther. 2012;50(7):510-3.

13. Rosner $\mathrm{MH}$. Cromolyn sodium: a potential therapy for uremic pruritus? Hemodial Int. 2006;10(2):189-92. 
14. Ye T, Tu W, Xu G. Hot bath for the treatment of chronic renal failure. Ren Fail. 2014;36(1):126-30.

15. Chen B, Zhan H, Chung M, Lin X, Zhang M, Pang J, Wang C. Chinese herbal Bath therapy for the treatment of knee osteoarthritis: meta-analysis of randomized controlled trials. Evid Based Complement Alternat Med. 2015; 2015:949172.

16. Bedi MK, Shenefelt PD. Herbal therapy in dermatology. Arch Dermatol. 2002; 138(2):232-42

17. Wojcikowski K, Johnson DW, Gobe G. Herbs or natural substances as complementary therapies for chronic kidney disease: ideas for future studies. J Lab Clin Med. 2006;147(4):160-6.

18. Higgins JP, Green S. Cochrane handbook for systematic reviews of interventions. Version 5.1.0[updated march 2011]: The Cochrane Collaboration; 2011. http://handbook-5-1.cochrane.org/.

19. Du W. Clinical observation on the treatment of uremia combined with pruritus of skin by Chinese herbal bath. Chin Commun Doctors. 2009;11(22):122.

20. Du MJ, Wen YW, Sun LH. Chinese herbal bath for treating 15 patients with uremic pruritus. Clin J Tradit Chin Med. 2004;16(02):126.

21. Gao JM, Ye JY. Clinical observation of 21 patients of uremic pruritus treated by antipruritic Chinese herbal bath. Clin Focus. 2012;27(03):257-8.

22. Guo XL, Tan JC, Wang G. Clinical observation of skin pruritus in hemodialysis patients treated by traditional Chinese herbal bath. China Modern Med. 2009;16(07):84-5.

23. Jia YL, Li XM, Du JH, Chang Y. Observation on curative effect of treating uremic pruritus with Shengshizhiyang tang. J Hebei Med Univ. 2012;33(12): 1434-6.

24. Jia YL, Li XM, Fei YL. Effect of Shengshizhiyang tang on uremia itchy skin. J Hainan Med Univ. 2012;18(05):646-647+650.

25. Lan SY. Analysis on the effect of Chinese herb medicated Bath in the treatment of cutaneous pruritus of maintenance hemodialysis. Guangming Tradit Chin Med. 2017;32(19):2791-3.

26. Lin YF. Clinical efficacy and safety analysis of Shengshizhiyang tang washing for treating patients with uremic pruritus. Chin J Tradit Med Sci Technol. 2014:21(z2):32-2

27. Shen $\mathrm{H}$. Clinical observation on the effect of Chinese herbal bath on relieving pruritus in hemodialysis patients. World Health Digest. 2014;25(25): 285-6.

28. Wang LY, Zhao JS, Luo M, wang FL, Yang M. Clinical observation of Chinese herbal bath for treating 27 patients with uremic pruritus. Chin Med Mod Dist Educ China. 2013;11(20):35-5.

29. Wen YW. Chinese herbal bath for treating 21 hemodialysis patients with uremic pruritus. J Emerg Tradit Chin Med. 2007;16(05):623.

30. Yao L. TCM formula granule washing for treating for 40 patients with uremic pruritus. China Pharmaceuticals. 2015:24(B11):65-6.

31. Yu YN, Wang LL, Wu XB. Curative effect observation of applying dispelling wind and dampness itching medicated Bath therapy for uremic pruritus. New Chin Med. 2017;49(12):64-6.

32. Zhang $C L$, Yu GZ, Gui $X Q$, Huang $C E$, Zhang XW. Experience of nursing uremic pruritus with Chinese medicine formula granule. Chin Med Mod Dist Educ China. 2014;12(20):128-9.

33. Zhang YL, Zhu HF, Wei SZ, Li J. Clinical observation of Chinese herbal bath for treating 78 patients with uremic pruritus. World Health Digest. 2012; 9(41):382-3.

34. Zhao H. Open Cou discharge turbidity dipping treatments for uremia itchy skin clinical research. Master's Degree. Nanjing: Nanjing University of Chinese Medicine; 2011.

35. Zheng J. Observation on the curative effect of Yangxuezhiyang mixture on uremia patients. Contmporary Med. 2016;22(24):160-1.

36. Ling YK. Chinese Materia Medica. Shanghai: Shanghai Science and Technology Press; 1984.

37. Gao XM. Advanced series of books for Chinese medicine: Chinese Materia Medica. Beijing: People's Medical Publishing House; 2000.

38. Gao XM. Science of Chinese traditional medicine. Beijing: China Press of Traditional Chinese Medicine; 2007

39. Liu LL, Duan J. A study about the elimination of uremia toxins by traditional Chinese medicine bath therapy from the theory of "opening ghost door". J New Chin Med. 2013;45(07):189-90.

40. Xiao XR. Drug bath therapy for uremia. Chin Health Care. 2009;03:22.

41. Chen SQ. Chinese herbal bath for treating 20 patients with uremia. Sichuan J Tradit Chin Med. 2003;21(02):47.
42. Lu XY, Zhang GY, Jia XT, Liang PY, Shi GM, Yang XR, Hu ML. Clinical study on treatment of chronic renal insufficiency with Chinese medicine bath. In: The sixth national conference on integrated Chinese and western medicine for kidney disease. Taiyuan: Chinese Association of Integrative Medicine: 2000.

43. Sun JG, Zhang RY. Chinese medicine bath for treating 20 patients with uremia. Zhejiang J Tradit Chin Med. 1994;3(3):134.

44. Sun XJ, Zhou CX. Study on the mechanism of drug bath therapy. J Jiangxi Univ Tradit Chin Med. 2007:19(05):25-6.

45. Cohen AD, Shalev R, Yaniv R, Shemer A. An open-label study of an herbal topical medication (QoolSkin) for patients with chronic plaque psoriasis. Sci World J. 2007;7:1063-9.

46. al-Tamer YY, Hadi EA, al-Badrani II. Sweat urea, uric acid and creatinine concentrations in uraemic patients. Urol Res. 1997;25(5):337-40.

47. Yosipovitch G, Reis J, Tur E, Blau H, Harell D, Morduchowicz G, Boner G. Sweat electrolytes in patients with advanced renal failure. J Lab Clin Med. 1994;124(6):808-12.

48. Falodun O, Ogunbiyi A, Salako B, George AK. Skin changes in patients with chronic renal failure. Saudi J Kidney Dis Transplant. 2011;22(2):268-72.

49. Sato K. The physiology, pharmacology, and biochemistry of the eccrine sweat gland. Rev Physiol Biochem Pharmacol. 1977;79:51-131.

50. Song C. A study on the effect of Chinese herbal bath on the quality of life of hemodialysis patients with chronic renal failure. Master's Degree. Nanjing: Nanjing University of Chinese Medicine; 2014

51. Jia YH, Liu P, Yang LB. Curative effects of Chinese herbal bath combined with Western medicine in treatment of chronic renal failure caused by chronic glomerulonephritis. Lab Med Clin. 2015;12(10):1374-1375+1377.

52. Wang CF, Xia Y. Clinical observation on the treatment of chronic renal failure with traditional Chinese medicine. Chin Commun Doctors. 2012; 14(21):218-9.

53. Zhou JL, Dong FX. Study on the treatment of uremic pruritus by traditional Chinese medicine bath. Zhejiang J Integr Tradit Chin W Med. 2004;14(01): 53-4.

54. Luurila OJ. The sauna and the heart. J Intern Med. 1992;231(4):319-20.
Ready to submit your research? Choose BMC and benefit from:

- fast, convenient online submission

- thorough peer review by experienced researchers in your field

- rapid publication on acceptance

- support for research data, including large and complex data types

- gold Open Access which fosters wider collaboration and increased citations

- maximum visibility for your research: over $100 \mathrm{M}$ website views per year

At $\mathrm{BMC}$, research is always in progress.

Learn more biomedcentral.com/submissions 